\title{
GENERALIZED DERIVATIONS ACTING AS HOMOMORPHISMS AND ANTI-HOMOMORPHISMS ON LIE IDEALS OF PRIME RINGS
}

\author{
Akhil Chandra Paul ${ }^{1}$ and Sujoy Chakraborty ${ }^{2}$ \\ ${ }^{1}$ Department of Mathematics, Rajshahi University, Rajshahi, Bangladesh \\ ${ }^{2}$ Department of Mathematics, Shahjalal University of Science and Technology, Sylhet, Bangladesh \\ Corresponding author: sujoy_chbty@yahoo.com
}

Received 02.12.2014 Accepted 18.08.2015

\begin{abstract}
Let $U$ be a non-zero square closed Lie ideal of a 2-torsion free prime ring $R$ and $f$ a generalized derivation of $R$ with the associated derivation $d$ of $R$. If $f$ acts as a homomorphism and as antihomomorphism on $U$, then we prove that $d=0$ or $U \subset Z(R)$, the centre of $R$.
\end{abstract}

Keywords: Prime ring, Lie ideal, Generalized derivation

\section{Introduction}

Let us consider $R$ to be an associative ring with centre $Z(R)$ throughout the article.

A ring $R$ is said to be 2-torsion free if $2 x=0$ with $x \in R$, then $x=0$. A ring $R$ is called a prime ring if for any $x, y \in R, x R y=0$ implies $x=0$ or $y=0$.

In a ring $R$, the symbol $[x, y]$ is known as the commutator of $x$ and $y$, which is defined by $[x, y]=$ $x y-y x$, where $x, y \in R$. Two useful basic commutator identities are:

$$
[x y, z]=x[y, z]+[x, z] y \text { and }[x, y z]=y[x, z]+[x, y] z .
$$

An additive subgroup $U$ of $R$ is said to be a Lie ideal of $R$ if $[u, r] \in U$ for all $u \in U$ and $r \in R$. A Lie ideal $U$ of $R$ is called a square closed Lie ideal if $u^{2} \in U$ for all $u \in U$.

An additive mapping $d: R \rightarrow R$ is said to be a derivation if $d(x y)=d(x) y+y d(x)$ for all $x, y \in R$. An additive mapping $f: R \rightarrow R$ is called a generalized derivation if there is a derivation $d: R \rightarrow R$ such that $f(x y)=f(x) y+y d(x)$ holds for all $x, y \in R$.

Let $S$ be a non-empty subset of $R$ and $f$ a generalized derivation of $R$. If $f(x y)=f(x) f(y)$ [resp. $f(x y)=f(y) f(x)]$ for all $x, y \in S$, then $f$ is said to act as a homomorphism [resp. as an antihomomorphism] on $S$.

The notion of generalized derivation was introduced by Bresar [4] and several characterizations of generalized derivation were obtained by B. Hvala [6] and T. K. Lee [7]. In [2], Bell and Kappe prove that if a derivation acts as a homomorphism and as an anti-homomorphism on a non-zero 
ideal $I$ of a prime ring $R$, then $d=0$. Asma, Rehman and Shakir [1] extend this result to a square closed Lie ideal, whereas Rehman [8] proves the same result for generalized derivations.

In this article, we extend the main result of [8] to square closed Lie ideals by using the similar arguments to get the following result.

Theorem 1.1 Let $U \neq 0$ be a square closed Lie ideal of a 2-torsion free prime ring $R$, and $f$ a generalized derivation of $R$ with the associated derivation $d$ of $R$.

(i) Iff acts as a homomorphism on $U$, then $d=0$ or $U \subset Z(R)$.

(ii) Iff acts as an anti-homomorphism on $U$, then $d=0$ or $U \subset Z(R)$.

\section{Main Results}

We begin with the following two lemmas (established earlier) which are needed to accomplish the desired proof of our Theorem 1.1.

Lemma 2.1 ([3], Lemma 4) Let $U \not \subset Z(R)$ be a Lie ideal of a 2-torsion free prime ring $R$ and $a, b \in R$ such that $a U b=0$. Then $a=0$ or $b=0$.

Lemma 2.2 ([3], Lemma 5) Let $U \neq 0$ be a Lie ideal of a 2-torsion free prime ring $R$ and $d \neq 0$ a derivation of $R$ such that $d(U)=0$. Then $U \subset Z(R)$.

The following useful result plays an important role to reach the goal.

Lemma 2.3 If $U \neq 0$ is a Lie ideal of a 2-torsion free prime $\operatorname{ring} R$ such that $[U, U]=0$, then $U \subset Z(R)$.

Proof. For all $u \in U$ and $x \in R$, we have

$$
[u,[u, x]]=0
$$

Replacing $x$ by $x y$ with $y \in R$, and using (1), we obtain

$$
\begin{aligned}
0 & =[u, x[u, y]+[u, x] y] \\
& =x[u,[u, y]]+[u, x][u, y]+[u, x][u, y]+[u,[u, x]] y \\
& =2[u, x][u, y] .
\end{aligned}
$$

Since $R$ is 2-torsion free, we get

$$
[u, x][u, y]=0
$$

for all $u \in U$ and $x, y \in R$.

Putting $y z$ for $y$ in (2) with $z \in R$, and using (2), we obtain

$$
[u, x] y[u, z]=0 \text { for all } u \in U \text { and } x, y, z \in R \text {. }
$$


Thus, we have $[u, x] R[u, z]=0$.

So, $[u, x]=0$ or $[u, z]=0$ for all $u \in U$ and $x, z \in R$ (by the primeness of $R$ ).

In both the cases, we see that $U \subset Z(R)$.

We are now in a position to prove our main result in the following way.

Proof of Theorem 1.1 Let us assume that $U \not \subset Z(R)$.

Since $U$ is a square closed Lie ideal, we have

$$
u v+v u=(u+v)(u+v)-u^{2}-v^{2} \in U \text { for all } u, v \in U .
$$

Also, we get $u v-v u \in U$ for all $u, v \in U$.

So, $2 u v \in U$ for all $u, v \in U$.

Therefore, $4(u v w)=2(2 u v) w \in U$ for all $u, v, w \in U$.

(i) If $f$ acts as a homomorphism on $U$, then we obtain

$$
\begin{aligned}
f(4 u v w) & =f(2(2 u v) w)=4 f(u v) w+4 u v d(w) \\
& =4(f(u) f(v) w+u v d(w))
\end{aligned}
$$

for all $u, v, w \in U$.

On the other hand,

$$
\begin{aligned}
f(4 u v w) & =f(2 u(2 v w))=4 f(u) f(v w) \\
& =4(f(u) f(v) w+f(u) v d(w))
\end{aligned}
$$

for all $u, v, w \in U$.

Comparing (3) and (4), and using the 2-torsion freeness of $R$, we get

$$
f(u) v d(w)=u v d(w),
$$

which yields

$$
(f(u)-u) v d(w)=0
$$

for all $u, v, w \in U$.

Thus, we have $(f(u)-u) U d(w)=0$ for all $u, w \in U$.

In view of Lemma 2.1, we obtain that

$$
f(u)-u=0 \text { for all } u \in U \text { or } d(w)=0 \text { for all } w \in U .
$$

If $d(w)=0$ for all $w \in U$, then by Lemma 2.2, we have $d=0$ or $U \subset Z(R)$. Since $U \not \subset Z(R)$, we get $d=0$. 
On the other hand, if $f(u)-u=0$ for all $u \in U$, then we have

$$
f(u)=u
$$

for all $u \in U$.

Replacing $u$ by $2 u v$ in (6) for $v \in U$, and using the 2-torsion freeness of $R$, we get

$$
u v=f(u v)=f(u) v+u d(v)=u v+u d(v) \text { for all } u, v \in U .
$$

Thus, we have $u d(v)=0$ for all $u, v \in U$.

Therefore, $U d(v)=0$ for all $v \in U$.

Since $[U, R] \subset U$, we obtain $[U, R] d(v)=0$ for all $v \in U$.

This yields, $U R d(v)=0$ for all $v \in U$.

Because $U \neq 0$ and $R$ is prime, we have $d(v)=0$ for all $v \in U$.

Thus, by Lemma 2.1, we get $d=0$ or $U \subset Z(R)$. The fact $U \not \subset Z(R)$ forces $d=0$.

(ii) Let us suppose that $f$ acts as an anti-homomorphism on $U$. Then we have

$$
f(u) v+u d(v)=f(v) f(u)=f(u v)
$$

for all $u, v \in U$.

Putting $2 u v$ in place of $u$ in (7), and using (7), we obtain

$$
u v d(v)=f(v) u d(v)
$$

for all $u, v \in U$.

Substituting $2 w u$ for $u$ in (8), we get

$$
w u v d(v)=f(v) w u d(v)
$$

for all $u, v, w \in U$.

Multiplying (8) by $w$ on the left, we have

$$
\operatorname{wuvd}(v)=w f(v) u d(v)
$$

for all $u, v, w \in U$.

Comparing (9) and (10), we obtain

$$
[w, f(v)] u d(v)=0
$$

for all $u, v, w \in U$.

In view of Lemma 2.1, we get

$$
[w, f(v)]=0 \text { for all } v, w \in U \text { or } d(v)=0 \text { for all } v \in U .
$$

If $d(v)=0$ for all $v \in U$, then by Lemma 2.2, we find $d=0$ or $U \subset Z(R)$, and hence $d=0$, since $U \not \subset Z(R)$. 
On the other hand, if

$$
[w, f(v)]=0
$$

for all $v, w \in U$, then upon replacing $v$ by $2 v w$ in (12), and using (12), we have

$$
v[w, d(w)]+[w, v] d(w)=0
$$

for all $v, w \in U$.

Again, substituting $2 u v$ for $v$ in (13) for $v \in U$, and using 2-torsion freeness of $R$, we get

$$
\begin{aligned}
0 & =u v[w, d(w)]+[w, u v] d(w) \\
& =u v[w, d(w)]+u[w, v] d(w)+[w, u] v d(w) \\
& =u(v[w, d(w)]+[w, v] d(w))+[w, u] v d(w) . \\
& =[w, u] v d(w), \text { by using }(13) .
\end{aligned}
$$

Thus, we obtain

$$
[w, u] U d(w)=0
$$

for all $u, w \in U$.

Applying Lemma 2.1 in (14), we find that

$$
d(w)=0 \text { for all } w \in U \text { or }[w, u]=0 \text { for all } u, w \in U .
$$

If $[w, u]=0$ for all $u, w \in U$, the in view of Lemma 2.3, it follows that $U \subset Z(R)$, which is a contradiction to the fact that $U \not \subset Z(R)$. So, we have $d(w)=0$ for all $w \in U$.

By using Lemma 2.2, we have $d=0$ or $U \subset Z(R)$.

Since $U \not \subset Z(R)$, we conclude that $d=0$.

\section{REFERENCES}

[1] A. Asma, N. Rehman and A. Shakir, (2003), On Lie ideals with derivations as homomorphisms and anti-homomorphisms, Acta Math. Hungar., 101(1-2) : 79-82.

[2] H. E. Bell and L. C. Kappe, (1989), Rings in which derivations satisfy certain algebraic conditions, Acta Math. Hungar., 53(3-4):339-346.

[3] Bergen, I. N. Herstein and J. W. Kerr, (1981), Lie ideals and derivations of prime rings, J. Algebra, 71: 259-267.

[4] M. Bresar, (1991), On the distance of the compositions of two derivations to the generalized derivations, Glasgow Math. J., 33 : 80-93.

[5] I. N. Herstein, (1969), Topics in Ring Theory, University of Chicago Press, Chicago.

[6] B. Hvala, (1998), Generalized derivations in prime rings, Comm. Algebra, 26(4): 1147-1166.

[7] T. K. Lee, (1999), Generalized derivations of left faithful rings, Comm. Algebra, 27(8) : 4057-4073.

[8] N. Rehman, (2004), On generalized derivations as homomorphisms and anti-homomorphisms, Glasnik Mat., 39(59) : 27-30. 